In rabbits, $9 \mathrm{~g}$ rectus femoris (RFM) muscles were grafted with tendon repair and with (1) nerves and blood vessels intact, (2) nerves intact and blood vessels anastomosed, or (3) nerves and blood vessels anastomosed. The influences of tendon, nerve, and vascular repair on the functional capabilities of grafts were compared 15-120 days after grafting. Data were collected on the mass, total protein content, oxidative capacity, maximum force development, and fatigability of grafts and control RFM muscles. When stabilized 90-120 days after grafting, mean values for the three types of grafts were not significantly different. Compared with values for control RFM muscles, each type of graft had significantly lower mean values for mass, total protein content, and maximum force, but the grafts were more resistant to fatigue. In RFM grafts, nerve and vascular repair do not contribute significantly to the impairments. Consequently, tendon repair appears to be responsible for the major functional deficits.

MUSCLE \& NERVE 11:745-751

\title{
NEUROVASCULAR-ANASTOMOSED MUSCLE GRAFTS IN RABBITS: FUNCTIONAL DEFICITS RESULT FROM TENDON REPAIR
}

PAUL J. GUELINCKX, MD, JOHN A. FAULKNER, PhD, and DAVID A. ESSIG, PhD

Following the ischemia of free grafting, skeletal muscles larger than $6 \mathrm{~g}$ become necrotic, are infiltrated with connective tissue, show little evidence of viable muscle, and develop no measureable force (for reviews, see Refs. 1 and 5). Transplantation with neurovascular anastomoses allows muscles larger than $6 \mathrm{~g}$ to be transplanted with litthe evidence of necrosis or excessive connective tissue ${ }^{15}$ the preservation of muscle mass, and the development of significant force. ${ }^{\gamma, 10,17}$ Despite the success of grafting muscles with neurovascular anastomoses, considerable variability is observed in the functional recovery of the grafts. When the

From the Departments of Physiology (Drs. Guelınckx and Faulkner) and Kinesiology (Dr Essig), the University of Michigan, Ann Arbor. MI

Acknowledgments: We thank Dr. Bruce M. Carlson for his assistance in the interpretation of histological sections The research was supported by program projecl grant DE 07687 from the National Institute of Dental Research and grant HL 34164 from National Heart, Lung, and Blood Institute Dr. Guelinckx's current address is Department of General Surgery. Division of Plastic Surgery and Microsurgery. The Catholic University of Leuven, Leuven, Belgium. Dr. Essig's current address is Department of Medicine. Section Cardiology. The University of Chicago. Chicago. IL.

Address reprint requests to Dr. Faulkner at the Department of Physiology, the University of Michigan. Ann Arbor. MI 48109-0622.

Accepted for publication July 15, 1987

$0148-639 \times / 1107 / 0745 \$ 0400 / 7$

(C) 1988 John Wiley \& Sons, Inc

maximum isometric tetanic force $\left(P_{\mathrm{o}}\right)$ of grafts made with neurovascular anastomoses is expressed as a percentage of the control value, the $P_{0}$, reported in different studies has varied from $25 \%{ }^{17}$ to $55 \%$ and within a single study from 39 to $74 \% .{ }^{\circ}$ For small free grafts in rats ${ }^{2.4}$ and cats, ${ }^{6}$ the functional recovery of grafts is also improved by grafting muscles with the nerve left intact. The rectus femoris (RFM) muscle graft in rabbits provides an animal model of grafting similar to the grafting models used clinically ${ }^{7}$ in terms of mass, single neurovascular pedicle, and variability of functional recovery. Our purpose was to evaluate during whole muscle grafting the relative contributions of the repair of the tendons, of the nerve, and of the blood vessels to the magnitude of the structural and functional deficits found in the grafts. We utilized the techniques of grafting with nerves and vasculature intact (NVI grafts), with nerves intact and vasculature anastomosis (NI-VA grafts), and nerve and vasculature anastomoses (NVA grafts) to clarify the contributions of each of these operative procedures to the deficits observed in stabilized grafts of RFM muscles in rabbits.

\section{material and methods}

Skeletal muscles were grafted in 43 healthy New Zealand white male rabbits (body mass $3.1 \pm 0.06$ 
$\mathrm{kg}, \overline{\mathrm{X}} \pm \mathrm{SE}$ ). Data were collected on 4 control RFM muscles from rabbits of similar body mass. The grafting procedures were performed under sterile conditions. Rabbits were anesthetized with a combination of phentanyl and fluanison I.M. $(0.5 \mathrm{mg} / \mathrm{g}$ body mass). Treatment of the rabbits during the operative and postoperative periods was in accordance with the Guiding Principles in the Care and Use of Animals of the American Physiological Society. Following the collection of data, the animals were killed by a lethal injection of pentobarbital sodium solution.

All RFM muscles were grafted orthotopically. The muscles were grafted bilaterally in four animals. In the remaining 39 animals, only the left RFM muscle was grafted. Of the 47 RFM muscles grafted, 8 were NVI grafts, 18 were NI-VA grafts, and 2I were NVA grafts. In each grafting operation, the RFM muscle was exposed and isolated, and the tendons of the muscle were severed. As few muscle fibers as possible were damaged, but satisfactory stumps were left at both the origin and the insertion. The neurovascular pedicle was dissected free under an operating microscope. For NVI grafts the muscle was lifted from its site without damage to the neurovascular pedicle and then replaced in the site. The blood flow to the NVI grafts was uninterrupted. For the NIVA grafts, the arterial and venous branches to the RFM muscle were clamped and severed without interrupting or stretching the branch of the femoral nerve to the RFM muscle. The muscle was lifted free and replaced immediately in its bed. The artery was repaired first, with 10-0 nylon suture using the "back wall first" technique for anastomosis. ${ }^{10}$ The vein was repaired between the femoral vein and the collecting site of the vena comitans and the vein on the anterior surface of the muscle. The blood flow to the NI-VA and NVA grafts was interrupted for 60-90 minutes during the vascular anastomosis but appeared fully restored when the clamps were removed from the vessels. For NVA grafts, perineural repair with I I-0 nylon suture was followed by vascular anatomosis and tendon repair. For each type of graft, the proximal and distal ends of the muscles were sutured securely to the fascia and tendon stumps with 5-0 nylon, and the fascia and skin were closed with interrupted stitches using 3-0 chromic catgut and 5-0 nylon suture, respectively. ${ }^{10}$

At 8 days after grafting, 3 animals with NVA grafts were anesthetized. The grafts were removed and placed in Bouin's solution for subsequent histological assessment of fiber degenera- tion. A complete cross section through the proximal and distal parts of each of the three grafts was removed and stained with hematoxylin $\&$ eosin. ${ }^{14}$ At 15 and 120 days, the mass, total protein content, succinic acid dehydrogenase (SDH) activity, and $P_{\mathrm{o}}$ of 4 NVI grafts, 4 NI-VA grafts, and 4 NVA grafts were compared (Table 1). Comparisons were also made of the mass. total protein content, SDH activity, and $P_{6}$ of NI-VA grafts and NVA grafts at $30(n=3$ in each group), $60(n=3)$, and $90(n=4)$ days after grafting. The $P_{\mathrm{o}}$ of the different types of grafts is plotted against time after grafting (Fig. 2). The resistance to fatigue of $4 \mathrm{NI}-\mathrm{VA}$ and 4 NVA grafts was compared with that of control RFM muscles 120 days after grafting (Fig. 3).

To measure the contractile properties, rabbits were anesthetized, and the RFM muscle or graft was isolated with the proximal tendon, nerve, and blood vessels intact. The distal tendon with patella and patellar ligament were connected to a Grass force transducer with 4-0 stainless steel wire. The nerve branch to the RFM muscle was isolated and divided under the inguinal ligament. The RFM muscle was maintained at $37^{\circ} \mathrm{C}$ in a skin pouch filled with mineral oil. A supramaximal electrical stimulus was applied to the nerve by means of stainless steel electrodes or directly to the muscle by means of electrodes inserted into the proximal and distal ends of the muscle. The stimulus duration was $0.2 \mathrm{msec}$. The muscle was adjusted to optimal length for force development $\left(L_{0}\right)$. Measurements were made of $P_{0}$ during both nerve and muscle stimulation. Fatigability of the RFM muscles and grafts was measured by continuous $3 \mathrm{~Hz}$ stimulation for 10 minutes. The twitch forces were plotted against time to assess changes in force with time. The ratio of potentiated twitch force over twitch force at 10 minutes was used as a fatigue index.

After measurement of contractile properties, the muscles were excised and weighed. For NIVA and NVA grafts, a section was removed from the proximal, middle, and distal part of the muscle for measurements of total protein content and SDH activity. Protein was assayed by the Lowry technique. " The SDH activity was assayed biochemically as a marker for mitochondrial oxidative capacity. SDH activity was assayed by the method described by Clark and Porteus. ${ }^{3}$

Statistical significance among the three types of grafts at 15 and 120 days was determined by a one-way analysis of variance. When significant $F$ ratios were obtained, statistical differences be- 
tween groups were determined by $t$-tests using the Welch-Aspin convention, which does not require equal sample sizes and assumes variancs to be unknown and not necessarily equal. ${ }^{12}$ The level of significance accepted a priori was $P \leq 0.05$. Data are presented as means \pm one standard error.

\section{RESULTS}

By 8 days after grafting, the histological sections from three NVA grafts showed survival of $95 \%$ or more of all muscle fibers (Fig. 1). At the proximal end of the muscle grafts, a small area of muscle fibers underwent degeneration apparently caused by repair of the tendon. At the distal end the area of damage was significantly smaller. Degeneration of skeletal muscle fibers was not evident in the central portion of the grafts, except for the occasional small area of damaged fibers just under the muscle fascia. Damage to peripheral fibers in the central portion of the graft was attributed to handling during the operation. A typical transition pattern of necrosis, degeneration with invasion of macrophages, myoblast formation, and restoration of muscle fibers was found in the areas of muscle damage (Fig. 1). Degeneration of fibers appeared to result from direct trauma rather than ischemia.

The vascularized RFM muscle grafts all maintained a bipennate structure, similar to that of the control RFM muscles. The mass of each of the three types of grafts was significantly lower than control values throughout the study (Table 1). The muscle masses of the NVI and NI-VA grafts remained at approximately $80 \%$ of the control value from 15 to 120 days after grafting, whereas the mass of the nerve-anastomosed grafts declined to $67 \% 15$ days after grafting, presumably due to denervation atrophy. The mass of the NVA grafts increased gradually to $80 \%$ at 90 days. For each type of graft, the total protein content followed a pattern similar to that of mass throughout the experimental period. Total SDH activity of both types of grafts demonstrated an initial drop in activity but recovered to control values by 90 days (Table 1).

For NVI and NI-VA grafts, no significant difference was observed between nerve and muscle stimulation at any of the test periods. For NVA grafts, nerve stimulation produced a significantly lower $P_{0}$ between 15 and 60 days after grafting (Fig. 2). After 90 days, no difference in $P_{\mathrm{o}}$ was evident between the two methods of stimulation. The $P_{o}$ of the 3 types of grafts increased steadily to reach an asymptote $90-120$ days after grafting at $62-71 \%$ of the control value (Fig. 2). The values for stabilized $P_{0}$ were not significantly different from one another.

During the fatigue test, the twitch responses of the RFM muscles and grafts to $3 \mathrm{~Hz}$ stimulation were similar (Fig. 3). Each response showed an initial potentiation by 1 minute, a decline in force, and, subsequently, a plateau. The RFM muscle potentiated to $173 \%$ of initial force, the NI-VA grafts to $158 \%$, and the NVA grafts to $120 \%$. The fatigue indices of $0.50 \pm 0.02$ for NI-VA grafts and $0.62 \pm 0.02$ for NVA grafts were significantly higher than the fatigue index of $0.35 \pm 1$ of the control RFM muscles (Fig. 3).

Table 1. The mass, total protein content, total SDH activity of control rectus femoris (RFM) muscles, and maximum isometric tetanic maximum force (expressed as a percent of the value for control RFM muscles) and of neurovascular-intact (NVI) grafts, nerve-intact vascular anastomosed (NI-VA) grafts, and neurovascular-anastomosed (NVA) grafts from 15 and 120 days after the grafting operation.

\begin{tabular}{|c|c|c|c|c|c|}
\hline & Mass (g) & $\begin{array}{l}\text { Total protein } \\
\text { content }(g)\end{array}$ & $\begin{array}{l}\text { Total SDH activity } \\
(\mu \mathrm{m} / \text { protein/hour }\end{array}$ & Maximum force $(\mathrm{N})$ & $\begin{array}{l}\text { Maximum force } \\
(\% \text { of control) }\end{array}$ \\
\hline $\begin{array}{l}\text { Control RFM muscle }(n=8) \\
15 \text { day grafts }\end{array}$ & $9.1 \pm 0.1$ & $1.61 \pm 0.09$ & $163.9 \pm 4.8$ & $41.2 \pm 0.4$ & 100 \\
\hline NVI grafts $(n=4)$ & $7.10 \pm 0.2$ & - & - & $15.7 \pm 0.8^{*}$ & 38 \\
\hline NI-VA grafts $(n=4)$ & $7.18 \pm 0.1$ & $1.45 \pm 0.17^{*}$ & $65.7 \pm 0.3^{*}$ & $15.5 \pm 0.4^{*}$ & 38 \\
\hline NVA grafts $(n=4)$ & $6.10 \pm 0.1^{*}$ & $0.94 \pm 0.4^{x} \dagger$ & $83.4 \pm 7.1^{*}$ & $16.6 \pm 0.6^{\star}$ & 40 \\
\hline \multicolumn{6}{|l|}{$90-120$ day grafts } \\
\hline NVI grafts $(n=4)$ & $6.7 \pm 0.1^{*}$ & - & - & $26.1 \pm 1.9^{\star}$ & 64 \\
\hline NI-VA grafts $(n=4)$ & $7.5 \pm 0.1$ & $1.16 \pm 0.97^{\star}$ & $188.9 \pm 4.8$ & $29.3 \pm 1.8^{*}$ & 71 \\
\hline NVA grafts $(n=4)$ & $7.3 \pm 0.1$ & $1.16 \pm 0.15^{\star}$ & $148.8 \pm 8.5 \dagger$ & $25.5 \pm 2.1^{*}$ & 62 \\
\hline
\end{tabular}

- Significant difference ( $P$ 0.05) between NVI, NI-NA, and NVA grafts and the control RFM muscle tSigniticant difference ( $P$ 0.05) between the NVA grafts compared with the NI-NA grafts. 

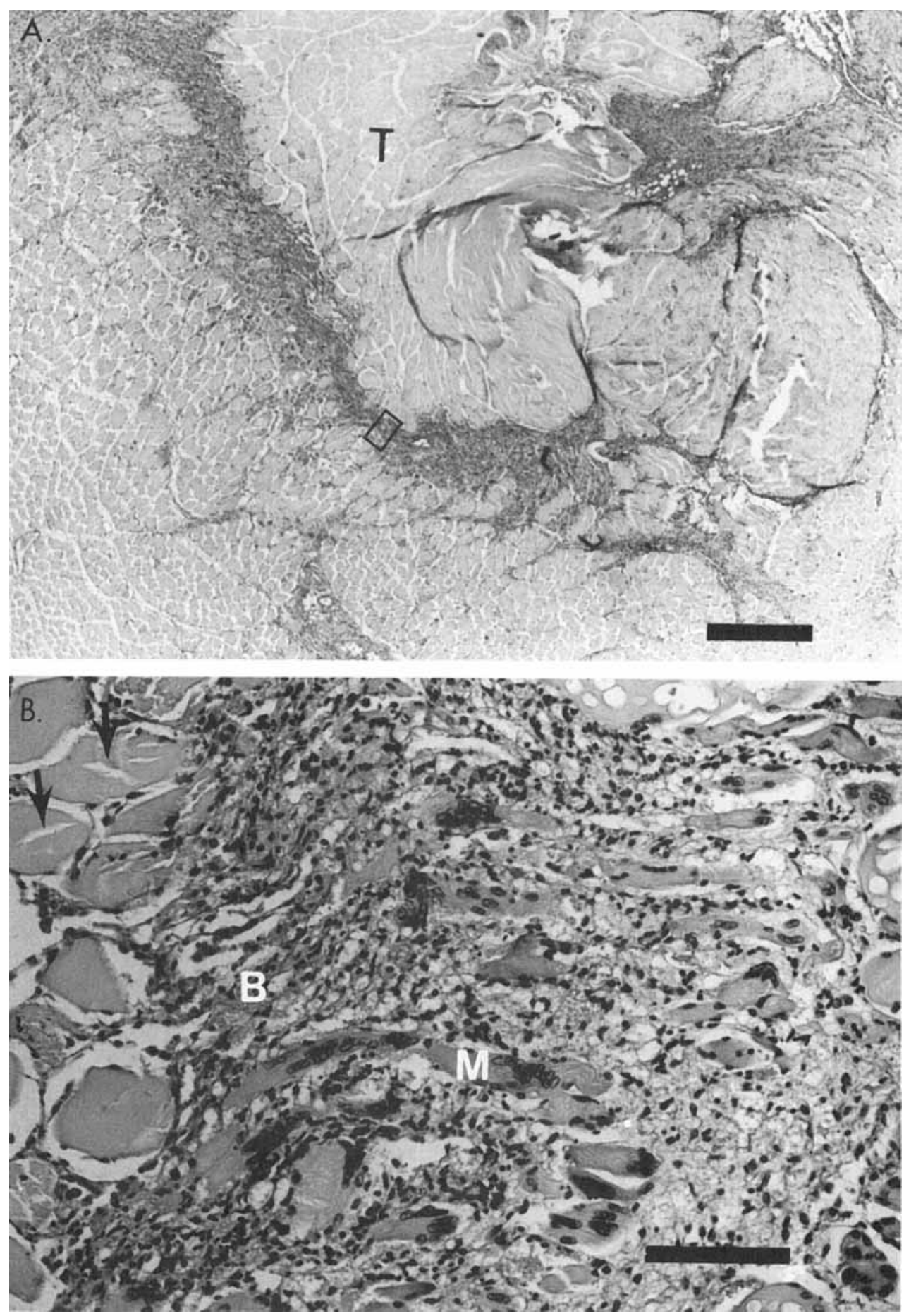

FIGURE 1. (A) By 8 days after grafting with microneurovascular repair, a small area of basophilia surrounds an area of necrosis in the region of the proximal tendon $(T)$. The basophilic area represents a zone of degeneration and regeneration of skeletal muscle fibers likely resulting from tenotomy and the surgical repair of the tendon. There is no overt histological evidence of injury to the fibers in the remainder of the cross section. A higher power view of the area enclosed by the rectangle is shown in $\mathbf{B}$. Hematoxylin \& eosin; bar = $0.2 \mathrm{~mm}$. (B) Higher power section of the area of basophilia $(B)$ viewed in $\mathbf{A}$. On the left side $(B)$ increased interstitial space is visible among degenerating muscle fibers. The degenerating fibers show characteristic transverse fissures (arrows). On the center and right of the section, most fibers have undergone sarcolysis. In this region, a cluster of regenerating myotubes $(\mathrm{M})$ is associated with interstitial phagocytic cells. Hemotoxylin \& eosin; bar $=100 \mathrm{~mm}$. 

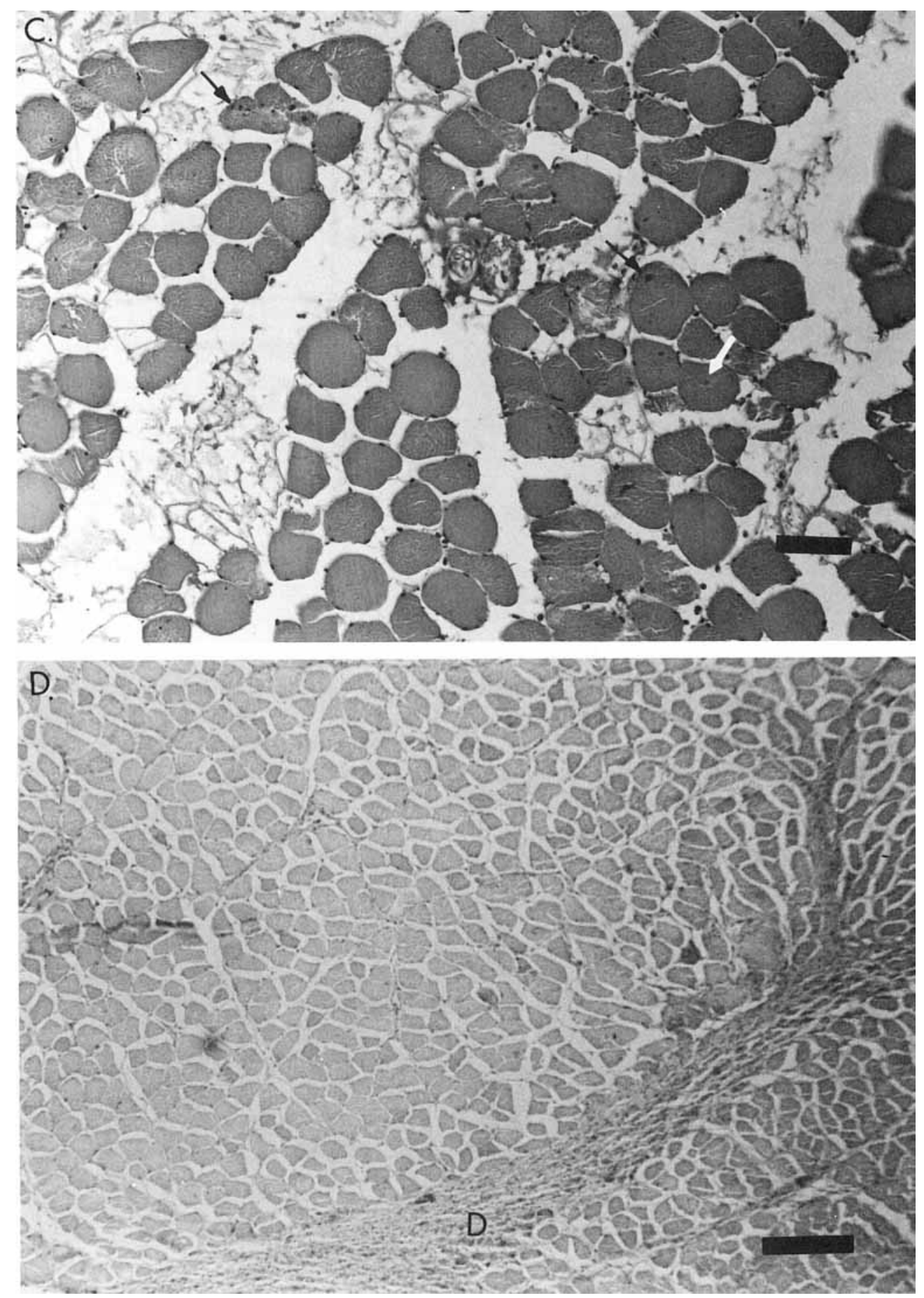

FIGURE 1, cont. (C) Section from the middle of the muscle belly in which most of the fibers appear undamaged except for a few that have central nuclei (arrows). The interstitial space is increased. Hemotoxylin \& eosin; bar $=0.1 \mu \mathrm{m}$. (D) Cross section through the lateral side of the midportion of an RFM graft shows an area of fiber degeneration (D). Under the epimysium, increased cellularity in the interfascicular connective tissue is associated with damage to skeletal muscle fibers along the lower right side of the fascicle. Hemotoxylin \& eosin; bar $=0.1 \mathrm{~mm}$. 


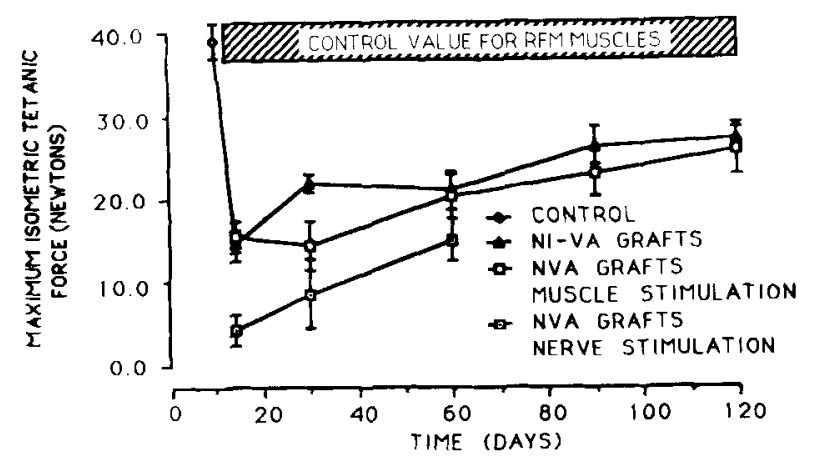

FIGURE 2. The maximum isometric tetanic force developed by nerve-intact vascular-anastomosed (NI-VA) grafts and neurovascular-anastomosed (NVA) grafts plotted against time after grafting. Both nerve and muscle stimulations are reported for the neurovascuiar-anastomosed grafts at the time periods when differences were significant $(\bar{X} \pm 1 \mathrm{SE})$.

\section{DISCUSSION}

The effect that grafting with neurovascular anastomoses has on the structure and function of skeletal muscle has not been described definitely for the period immediately after grafting. Based on histological evidence, Prendergast et al. ${ }^{15}$ concluded that the majority of skeletal muscle fibers survived in grafts made with neurovascular anastomoses. ${ }^{15}$ Our estimate of the survival of approximately $95 \%$ of the skeletal muscle fibers in the NVA grafts is in good agreement with the observations of Prendergast and his associates. ${ }^{15}$ In our study, degeneration and regeneration of fibers were observed primarily near the cut ends of the graft at the site of the tendon repair. More muscle fiber damage occurred at the proximal end than at the distal end of the muscle because of the broader muscular origin of the muscle on the pelvis. The fibers that degenerated likely sustained direct injury as a result of the opcrative procedure. The RFM muscle is bipennate, and liber lengths are $34 \%$ of the muscle length. Consequently, only a small proportion of the fibers were exposed to the trauma of cutting and suluring during grafting. The survival of fibers and observations during the grafting operation indicate that blood flow was uninterrupted to most of the mass of the NVI grafts and rapidly restored to most of the fibers in the NI-VA and NVA grafts.

The best single measure of the functional capabilities of grafts is the $P_{0}{ }^{5,6}$ The $P_{0}$ has been used to cvaluate the functional recovery of both free $1,2,5,6,16$ and vascularized ${ }^{8-10,17}$ grafts. Free grafis of extensor digitorum longus muscles in cats require 180 days for $P_{\mathrm{o}}$ to achieve stability. ${ }^{6}$ The shorter time (90 days) required for stabiliza-

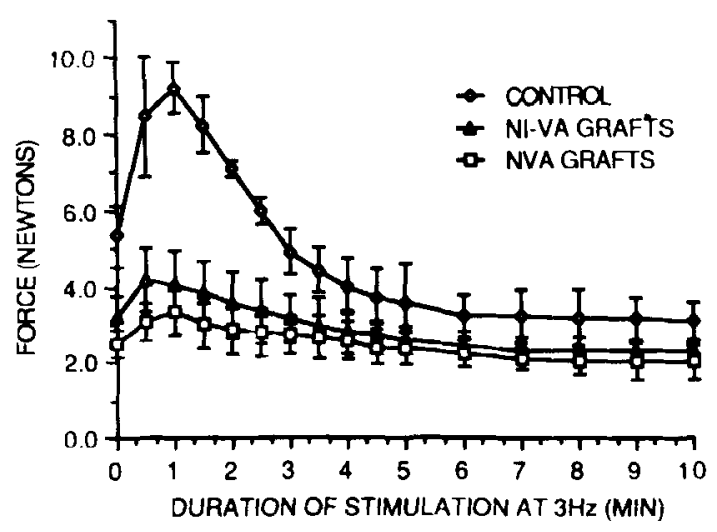

FIGURE 3. The twitch force developed by control RFM muscles, nerve-intact vascular-anastomosed (NI-VA) grafts, and neurovascular-anastomosed (NVA) grafts plotted against time of stimulation. Stimulation was at $3 \mathrm{~Hz}$. An initial potentiation, a decline in twitch force, and a plateau in twitch force are evident in each of the curves.

tion of the $P_{\mathrm{o}}$ of vascularized grafts compared with free grafts is likely due to the survival of the majority of the fibers in vascularized grafts ${ }^{15}$ and the degeneration and regeneration of fibers in the free grafts. ${ }^{1,5,14}$ The deficit of about $60 \%$ in the $P_{o}$ of each of the 3 types of grafts in rabbits $8-14$ days after grafting indicates a much greater loss of force than would be expected based on the histological evidence of injury. In a mouse extensor digitorum longus muscle, tenotomy and repair of the distal tendon with no damage to muscle fibers resulted in a $25 \%$ loss of $P_{6}, 3-5$ days after the operation. ${ }^{13}$ Consequently, a deficit in $P_{0}$ may occur even in the absence of direct injury to skeletal muscle fibers. The mechanism responsible for the deficit in $P_{o}$ is unknown.

The stabilization of the $P_{0}$ of the NVA grafts at $62 \%$ of the control value for RFM muscles indicates the recovery of a slightly greater relative $P_{\text {。 }}$ than has been reported for previous RFM grafts made with neurovascular anastomoses. ${ }^{8,10,18}$ The functional deficit in $P_{o}$ is associated with a $20 \%$ decrease in muscle mass and a $30 \%$ decrease in the content of total protein. Increases in connective tissue and volume of interstitial space are also possibilities. ${ }^{16}$ The lack of any major structural or functional difference among the three types of grafts indicates that the deficits in the grafts compared with control RFM muscles cannot be attributed to either the repair of the nerves ${ }^{9}$ or of the blood vessels. We conclude that the tenotomy and the repair of the tenotomy constituted the major cause of the long-term (120 day) deficit following grafting of $9 \mathrm{~g}$ muscles in rabbits. This conclusion 
differs from the observation of a complete recovery of the control value for $P_{0}$ by 30 days following tenotomy and repair of $8 \mathrm{mg}$ muscles in mice. $^{13}$

Although the mean deficits for the $P_{o}$ of stabilized NVI, NI-VA, and NVA grafts were not different, the NVA grafts showed much greater variability than NVI and NI-VA grafts. The range of deficits in $P_{\mathrm{o}}$ for NVA grafts was from 27 to $57 \%$, whereas NVI and NI-VA grafts ranged from 24 to $36 \%$. The increased range of deficits for NVA grafts is likely the result of variability in the success of the nerve anastomoses. ${ }^{9}$ Our range of deficits for the $P_{o}$ of NVA grafts of RFM muscles in rabbits are in excellent agreement with the range reported by Frey et al. ${ }^{8}$ of $26-61 \%$ for the same muscle and species.

The pattern of a greater resistance to fatigue in vascularized grafts compared with control muscles is similar to the greater resistance to fatigue in small $100 \mathrm{mg}$ free grafts in rats ${ }^{4}$ but significantly different from the increased fatigability observed for large $3 \mathrm{~g}$ free grafts in cats. ${ }^{6}$ The mechanism responsible for a change in the fatigability of muscles is not clear in any of these models. Compared with free muscle grafts, the greater $P_{0}$ and the greater resistance to fatigue of the vascularized grafts provide significant advantages for the restoration of the functional capabilities of injured or paralyzed tissues.

\section{REFERENCES}

1. Carison BM, Faulkner JA: The regeneration of skeletal muscle fibers following injury: a revicw. Med Sci Sports Exercise 15:187-198, 1983

2. Carlson BM, Hník $P$, Tŭcek $S$, Vejsada $R$, Bader $D$, Faulkner JA: Comparison between grafts with intact nerves and standard free grafts of the rat extensor digitorum longus muscle. Physiol Bohemoslov 30:505-513, 1981.

3. Clark B, Porteus JW: Determination of succinic acid by an enzymatic method. Biochem $J$ 93:21-30,1966.

4. Faulkner JA, Carlson BM: Contractile properties of standard and nerve-intact muscle grafts in the rat. Muscle Nerve 8:413-418, 1984

5. Faulkner JA, Côté C. Functional deficits in skeletal muscle grafts. Fed Proc 45:1466, 1986

6. Faulkner JA, Markley JM Jr, McCully KK, Waters CR, White TP: Characteristics of cat skeletal muscles grafted with intact nerves or with anastomosed nerves. Exp Neurol $80: 682-696,1983$.

7. Frey M, Freilinger G: 2nd Vienna Muscle Syposium Proceedings Vienna, Facultas Austria, 1986, pp 1-386.

8. Frey M, Gruber H, Havel M, Steiner E, Freilinger G: Experimental free muscle transplantation with microneurovascular anastomoses. Plast Reconstr Surg 71:689-701, 1983.

9. Frey M, Gruber J, Holle J, Freilinger G: An experimental comparison of the different kinds of muscle innervation: nerve suture, nerve implantation and muscular neurotization. Plast Reconstr Surg 69:656-667, 1983.

10. Guelinckx PJ, Dom R, Bex M, Boeckx WD, Gruwez JA: Rectus femoris muscle grafts performed with and without vascular anastomosis: an experimental study in the rabbit. Br I Plast Surg 37:584-595, 1984.

11. Lowry O, Rosebrough M, Farr A, Randall K: Protein measurement with the Folin phenol reagent. $J$ Biol Chem 193:264-275, 1951 .

12. Marascuilo LA: Statistical Methods for Behavioral Science Research. San Francisco, McGraw Publishers, 1971, pp $312-314$.

13. McCully KK, Faulkner JA: Injury to skeletal muscle fibers of mice following lengthening contractions. I Appl Physiol 59:119-126, 1985 .

14. Mufti SA, Carlson BM, Maxwell LC, Faulkner JA: The free grafting of entire limb muscles in the cat: morphology. Anat Rec 188:417-430, 1977.

15. Prendergast FJ, McGeachie JK, Edis RH, Allbrook D: Whole-muscle reimplantation with microneurovascular anastomoses. Ann R Coll Surg Engl 59:393-400, 1977.

16. Segal SS, White TP, Faulkner JA: Architecture, composition, and contractile propertics of rat soleus muscle grafts. Am J Physiol 19:C474-C479, 1986.

17. Terzis JK, Sweet RC, Dykes RW, Williams HB: Recovery of function in free muscle transplants using microneurovascular anastomoses. J Hand Surg 3:37-59, 1978. 